\title{
Hiccups and breathing in human fetuses
}

\author{
Mary Pillai, David James
}

\begin{abstract}
Serial recording in 45 low risk fetuses throughout the second and third trimesters showed that hiccups were the predominant diaphragmatic movement before 26 weeks' gestational age and that there was a significant negative correlation with gestational age. There was a pronounced reduction between 24 and 26 weeks, which was the result of a decrease in the number of episodes of hiccups rather than a change in the duration of episodes. In contrast, fetal breathing was positively correlated with gestational age, the greatest increase in breathing occurring between 26 and 32 weeks' gestation. This was the result of both an increase in the number and duration of episodes. From the time that rest-activity cycles of behaviour could be determined in recordings, both breathing and hiccups were dependent on behavioural state or cycle, occurring predominantly during active episodes. This association between quiet and active behaviour and breathing did not alter with increasing gestational age, and the variables in fetal behavioural state became increasingly closely linked.
\end{abstract}

The importance of prolonged and repeated recording, and also the need to take account of other variables in fetal behaviour, before any sinister conclusions can be drawn about the absence of fetal breathing is emphasised.

The human fetus makes a number of different diaphragmatic movements. With the notable exception of fetal breathing, however, there are virtually no data on these. Other types of diaphragmatic movement that have been described include sighs, hiccups, and gasping. Of these fetal hiccups are the most notable, but in contrast to breathing they have received scant attention and knowledge of the physiological mechanisms underlying their control and the part they play in fetal development is lacking.

Since the recognition that intermittent breathing activity is a normal feature of fetal life with a tendency to be stimulated by carbon dioxide and inhibited by hypoxia, ${ }^{12}$ many data have been published on fetal breathing in experimental animals using invasive techniques, especially in fetal lambs. With the development of non-invasive methods of fetal observation, interest in fetal breathing extended to it being used as an indicator of wellbeing in human fetuses. $^{34}$ Suppression of fetal breathing has been suggested to be an early predictor of fetal infection in patients with ruptured membranes, ${ }^{5}$ as a predictor of true preterm labour in pregnancies complicated by preterm contractions ${ }^{6}$ and as a predictor of the development of pulmonary hypoplasia in fetuses at high risk. ${ }^{78}$

All this has occurred despite little published longitudinal data about human fetuses, particularly in normally developing low risk fetuses, and so it is not surprising that those few studies in which fetal breathing in pathological circumstances has been matched with that in normal control fetuses, ${ }^{10}$ the results conflict with observations of fetal breathing in pathological circumstances only. ${ }^{7}$

The aims of this study were to document longitudinally, in normally developing low risk human fetuses, developmental trends in fetal breathing and hiccups, their association with developing patterns of fetal behaviour, and the range of individual variations.

Patients and methods

Women with no history of hypertension, renal disease, or diabetes and who had previously delivered at least one healthy normally grown infant at full term, and women having their first babies who had similar medical histories were invited to participate in a longitudinal study of multiple fetal variables. All those recruited smoked less than 10 cigarettes/day and each had a viable singleton pregnancy confirmed by ultrasound during the first 16 weeks of pregnancy.

Twenty one primigravidas and 24 multigravidas were recruited. Recordings were started between 14 and 18 weeks' gestation and repeated at monthly intervals until delivery. Fetal diaphragmatic movements and limb, body, and eye movements were detected with real time ultrasound and recorded polygraphically at a paper speed of $3 \mathrm{~cm} /$ minute. The fetal heart rate was also recorded ultrasonically using a standard fetal heart rate monitor (Hewlett Packard $8040 \mathrm{~A}$ ) with an output to the polygraph to allow synchronising of all variables. During most of the second trimester, recordings were made with a single scanner (Siemens Sonoline SLl with a $3.5 \mathrm{MHz}$ transducer), but in the last trimester two scanners were used, one giving a parasagittal view of the fetus's face (Siemens Sonoline SLl with a $3.5 \mathrm{MHz}$ transducer) and the other giving a transverse view of the fetus's abdomen (Toshiba SAL 20A with a $2.4 \mathrm{MHz}$ transducer). Fetal eye, somatic, and mouthing movements, and breathing and hiccups, were recorded on separate channels of the polygraph (Hewlett Packard 7754A) with mulitple hand held push buttons.

All recordings were made by a single observer 
(MP) in a quiet darkened room with the mother seated in a comfortable semirecumbent position, for which we found a Parker Knoll chair most suitable.

Recordings were undertaken between 0900 and 1800 , and continued for $90-100$ minutes. Within individual subjects recordings were repeated at the same time of day and at the same interval after meals, and the four women who smoked did not do so during, or for two hours before, recording. All participants were outpatients with no risk factors known to affect fetal behaviour or breathing.

The following definitions were used:

Fetal breathing: contraction of the diaphragm resulting in downward and outward displacement of the abdominal contents and inward displacement of the thorax. All episodes of fetal breathing were counted providing they comprised a minimum of four consecutive breaths sustained for at least five seconds. All episodes in which the breath to breath interval lasted for six seconds or more were excluded. ${ }^{11}$ Isolated diaphragmatic movements were not counted.

Fetal hiccups: characterised by more forceful and regular diaphragmatic contractions at a slower rate than fetal breathing. All episodes of hiccups comprising a minimum of five hiccups in 30 seconds, and sustained for 30 seconds, were counted.

Rest-activity cycles: a relatively high base rate of fetal movements interrupted by a period or periods of quiescence lasting six minutes or more (see below).

Behavioural states: stable association of heart rate pattern and the condition of eye movements and somatic movements for intervals of at least three minutes duration with simultaneous transition (within three minutes) of all three variables at times of transition between states. ${ }^{12}$

Statistical analysis of the data was by analysis of variance and the paired Student's $t$ test. Approval for the study was obtained from the hospital's research and ethics committee.

\section{Results}

A total of 268 recordings was made (mean 6.0 , range $4-7 /$ fetus) and the total recording time was $402 \cdot 4$ hours.

\section{BREATHING}

Before 26 weeks' gestation 105 recordings were made and these were characterised by a low percentage of fetal breathing movements (median $1 \cdot 4 \%$, range $0-26 \%$ ), with no breathing at all observed during $45(43 \%)$ of the recordings. Between 26 and 30 weeks' gestation there was a pronounced increase in the percentage of breathing with a continuing upward trend until 36 weeks' gestation (fig 1). The longest sustained intervals of fetal breathing showed a similar upward trend, but peaked $2-4$ weeks earlier (fig 2).

HICCUPS

Fetal hiccups showed a different developmental course, being significantly more common before

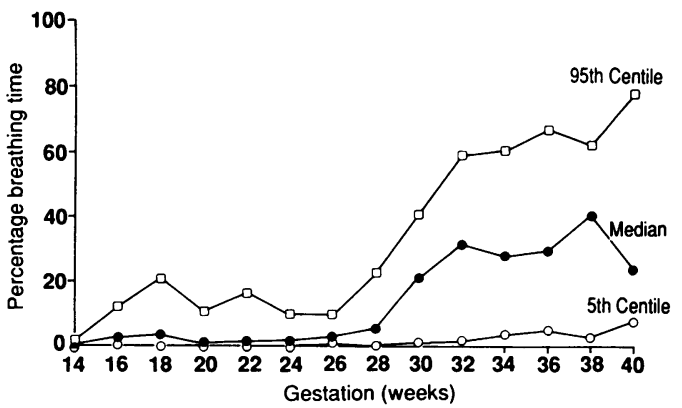

Figure 1 Median and centile values for the percentage of time fetal breathing was observed during 100 minute observation intervals every four weeks between 14 weeks and full term in 45 healthy fetuses.

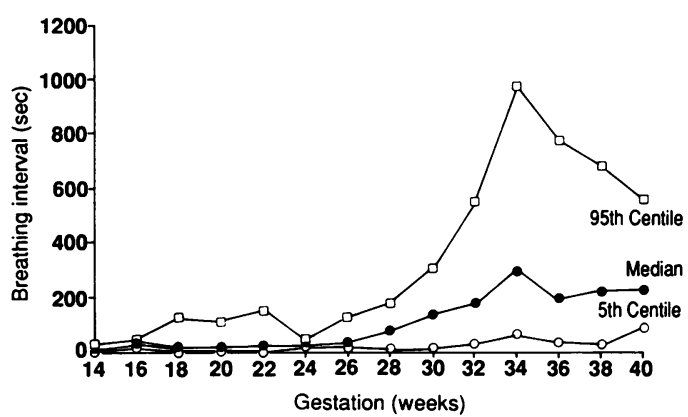

Figure 2 Median and centile values for the longest sustained intervals of fetal breathing (secs) in 45 healthy fetuses between 14 weeks and full term.

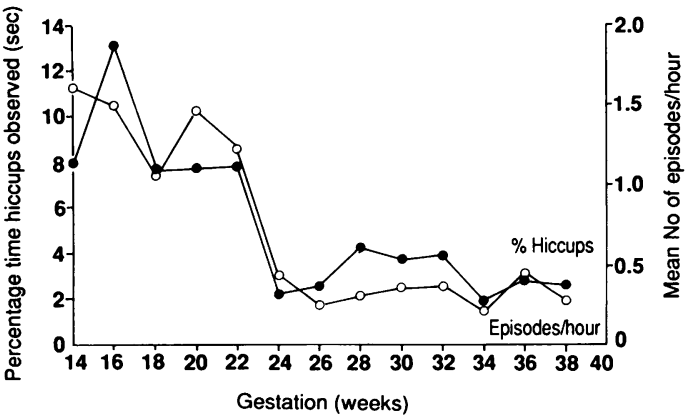

Figure 3 Mean percentage of time during which hiccups were observed $(r=-0 \cdot 33)$, and the mean number of episodes of hiccups/hour $(r=-0.47)$ in 45 healthy fetuses between 14 weeks and full term.

24 weeks' gestation than subsequently $(\mathrm{p}<0.0001)$, and this was solely because there were more episodes of hiccups before 24 weeks' gestation (fig 3). We did not find any developmental trends in the duration of episodes of hiccups after 18 weeks' gestation (fig 4). There was no apparent association between patterns of hiccups and breathing.

INDIVIDUAL FETAL VARIATION

With the exception of the period 32-36 weeks' gestation there were individual fetuses in which no breathing at all was observed during recording at all gestational ages. Similarly, with the exception of 33-34 weeks' gestation there were individual fetuses in which no intervals of breathing were sustained for 30 seconds, and at all 
$\mathrm{Sec}$

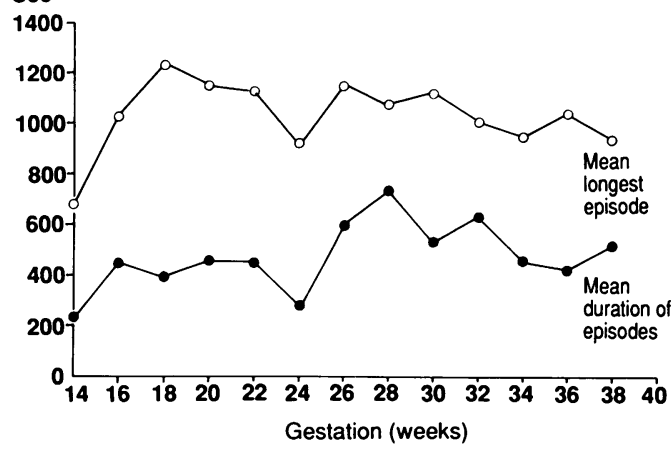

Figure 4 Mean duration of episodes of hiccups and mean duration of longest observed episode between 14 and 40 weeks gestation.

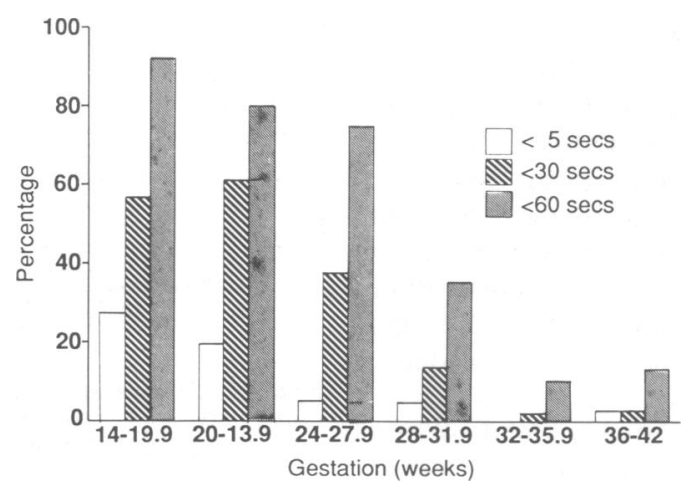

Figure 5 Percentage of recordings of $<5$ secs, $<30$ secs, and $<60$ secs of sustained fetal breathing in 260 recordings on 45 fetuses, with each fetus represented no more than once in each 4 week age group.

gestational ages there were fetuses in which no intervals were sustained for 60 seconds (fig 5). Breathing was observed during at least two recordings of each individual fetus. There was also considerable individual variation in hiccups, with several fetuses having no episodes in any of their recordings, and one had one episode or more during every recording.

\section{ASSOCIATION WITH BEHAVIOURAL STATES AND CYCLES}

With advancing gestational age there was a significant increase in the longest intervals of complete quiescence $(p<0.001)$, so that alternating cycles of rest and activity became more apparent. Using the arbitrary definition of six minutes for the minimum duration of a rest cycle, rest-activity cycles were rarely observed before 24 weeks' gestation, but by 29 weeks they were present in over $80 \%$ of recordings. Fixed and recurring association of body movements, eye movements, and fetal heart rate patterns into well defined fetal behavioural states were first observed in one fetus at 33 weeks' gestation and were present in over $80 \%$ of recordings by 36 weeks. ${ }^{12}$

Throughout the range of gestation studied, fetal breathing movements were significantly more common in the active cycles $(p<0.001)$ (fig 6), but within individual fetuses this find-

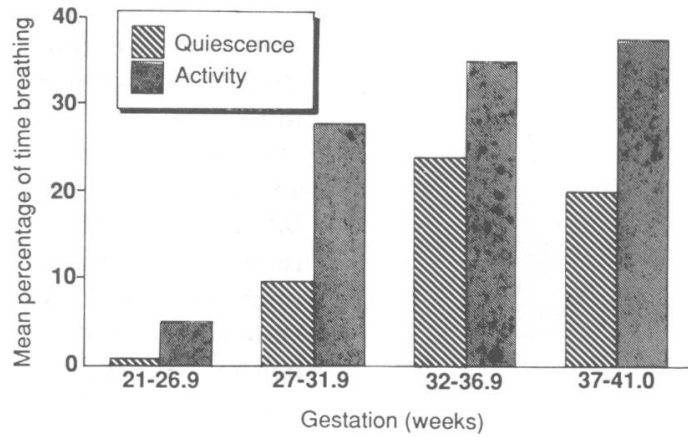

Figure 6 Mean percentage of time breathing was observed during rest and activity during only those recordings containing rest activity cycles (137 of 267 recordings). The differences between rest and activity were significant $(p<0.001$, paired t test $)$.

ing was not consistent, with six of the 137 recordings that contained rest and activity cycles $(4 \%)$ containing more breathing during quiescence than during the active cycles.

The onset of fetal hiccups was only observed during active cycles. In two recordings hiccups continued for several minutes in quiescence when a transition between cycles occurred shortly after their onset, but otherwise hiccups did not occur during periods of quiescence.

\section{FETAL OUTCOME}

All mothers delivered between 37 and 42 weeks' gestation. All one minute Apgar scores were 5 or more, and five minutes scores were 7 or more. The mean (SD) mean birth weight was 3532 (435) $g$ (range 2700-4650). One infant was admitted to the neonatal unit for investigation of pyrexia that settled without treatment, and one other infant died suddenly and inexplicably at 8 weeks. Further careful retrospective examination of the recordings of this infant did not uncover any differences compared with the group as a whole. All other infants were between 9 months and 2 years of age at the time of writing, and developing normally.

\section{Discussion}

The onset of both hiccups and breathing have been described during the first trimester, roughly one to two weeks after the development of the diaphragm ${ }^{13}$; their development thereafter, however, differs considerably, fetal hiccups being the predominant type of diaphragmatic movement until 26 weeks' gestation, after which fetal breathing predominates. This suggests that the centres that control fetal breathing are not only different, but also probably more complex, than those that control hiccups.

The early appearance of both types of activity suggests their ontogeny may depend only on a simple reflex, but the subsequent developmental course of breathing and experimental destructive lesions in fetal lambs shows that the development of higher centres has a profound influence on fetal breathing. ${ }^{14}$ 15 Such data about the developmental trends and influence of higher centres on hiccups is lacking, but our observations suggest that if subsequent develop- 
ment of the brain does influence hiccups the effect may well be inhibitory. Anecdotal evidence that supports this is our observation of hiccups continuing in compromised fetuses long after other types of normal behaviour, including breathing, have ceased (unpublished observations).

Van Woerden et al analysed hiccups in a cross sectional study of healthy fetuses born at full term. ${ }^{16}$ The frequency of episodes and percentage of recording time for hiccups were the same as we observed at comparable periods of gestation, but in contrast to our findings they found no specific association with behavioural states.

The data of Wigglesworth and Desai provided convincing evidence that breathing movements play an important part in the development of fetal lungs. ${ }^{17} 18$ Patrick et al have previously documented fetal breathing in the last 10 weeks of pregnancy, but their data are from single rather than serial studies. ${ }^{11} 19$ They drew attention to the fact that prolonged episodes of apnoea are characteristic of healthy human fetuses. Our data show that this is particularly so before 30 weeks' gestation whenduring prolonged recording-more than $40 \%$ of healthy fetuses have no breathing, and about $60 \%$ do not sustain breathing continuously for 30 seconds. This finding is important in relation to the observation of breathing in fetuses with prematurely ruptured membranes. The risk of pulmonary hypoplasia is greatest where the membranes rupture before 26 weeks' gestation and much less when they rupture after this time. Blott $e t a l^{7}$ and Blott and Greenough ${ }^{8}$ suggested that observation of breathing in the human fetus could be used to predict the development of pulmonary hypoplasia in fetuses at risk, but breathing movements are significantly less likely to be seen in normal healthy fetuses before 26 weeks' gestation thus raising questions about the specificity of this test.

Nijhuis $e t a l^{20}$ and van Vliet $e t a l^{21}$ were the first to study the association between behavioural states and breathing in human fetuses. They reported significantly more episodes of breathing during periods of activity than of quiescence between 32 weeks' gestation and full term, and that the linkage of breathing and other behavioural state variables did not alter with the emergence of behavioural states. Our data are in agreement with both these findings, but in addition show that the association between breathing and activity is present well before 32 weeks' gestation, though this association is not found consistently in all fetuses.

Experimental data on fetal lambs have shown that transition from the rapid eye movement to the non-rapid eye movement state is associated with a $30 \%$ reduction in cerebral oxygen and glucose metabolism. ${ }^{22}$ Thus in the short term the alteration in behavioural state may provide an important protective response to hypoxic or other stresses. It is possible therefore that the suppression of fetal breathing associated with fetal infection and preterm labour may be secondary to a change in state rather than primary suppression.
The main clinical implications of these data are, firstly, that they emphasise the need for prolonged and repeated recording of breathing activity before any sinister conclusions can be drawn. Secondly, during the last trimester fetal breathing is cycle or state dependent (being significantly less likely during episodes of quiescence) so that studies of the influence of complications of pregnancy on fetal breathing need also to take account of other variables of behaviour. Thirdly, in contrast to fetal breathing, hiccups predominate in immature fetuses and as yet there is no evidence to support their being a useful indicator of fetal health.

This study was funded by Action Research for the Crippled Child.

1 Dawes GS, Fox HE, Leduk BM, Liggins GC, Richards JS. Respiratory movements and rapid eye movement sleep in the foetal lamb. F Physiol 1972;220:119-43.

2 Boddy K, Davies GS. Fetal breathing. Br Med Bull 1975;31: 3-7.

3 Platt LD, Manning FA, LeMay M, Sipos L. Human fetal breathing movements: relationship to fetal condition. $A m$ f Obstet Gynecol 1978;132:514-8.

4 Manning FA, Platt LD. Monitoring the fetus using fetal breathing movements. Clin Obstet Gynaecol 1979;6:335-41.

5 breathing movements. Clin Obstet Gynaecol 1979,6:335-41. intzileos AM, Campbell WA, Nochimson DJ, Connolly ME, Fuenfer MM, Hoehn GJ. The fetal biophysical profile in patients with premature rupture of the membranes - an
early predictor of fetal infection. Am $\mathcal{F}$ Obstet Gynecol 1985; 152:510-6.

6 Agustsson P, Patel N. The predictive value of fetal breathing movements in the diagnosis of preterm labour. BrF Obste Gynaecol 1987;94:860-3.

7 Blott M, Greenough A, Nicolaides KH, Moscoso G, Gibb D. Fetal breathing movements as predictor of favourable pregnancy outcome after oligohydramnios due to membrane rupture in second trimester. Lancet 1987;ii:129-31.

8 Blott $M$, Greenough A. Oligohydramnios in the second trimester of pregnancy, fetal breathing and normal lung growth. Early Hum Dev 1988;17:37-40.

9 Fox HE, Moessinger AC. Fetal breathing movements and lung hypoplasia: preliminary human observations. Am 7 Obstet Gynecol 1985;151:531-3.

10 Moessinger AC, Fox HE, Higgins A, Rey HR, Al Haideri M. Fetal breathing movements are not a reliable predictor of continued lung development in pregnancies complicated by contigohydramnios. Lancet 1987;ii:1297-9.

11 Patrick J, Campbell K, Carmichael L, Natale R, Richardson B. A definition of human fetal apnea and the distribution B. A definition of human fetal apnea and the distribution
of fetal apneic intervals during the last ten weeks of of fetal apneic intervals during the last ten weeks

12 Nijhuis JG, Prechtl HFR, Martin CB Jr, Bots RSGM. Are there behavioural states in the human fetus? Early Human Dev 1982;6:177-95.

13 De Vries JIP, Visser GHA, Prechtl HFR. The emergence of fetal behaviour. I. Qualitative aspects. Early Hum Dev 1982;7:301-22.

4 Johnson $P$. In lambs fetal breathing in slow wave sleep becomes inhibited. In: Von Euler C, Lagercrantz $\mathrm{H}$, eds. Central nervous control mechanisms in breathing. Oxford: Pergamon Press, 1978:337-51.

15 Dawes GS, Gardner WN, Johnston NM, Walker DW. Breathing in fetal lambs; the effect of brain stem section. f Physiol (Lond) 1983;335:535-53.

16 Van Woerden EE, Van Geijn HP, Caron FJM, Mantel R, Swarties JM, Th Arts NF. Fetal hiccups; characteristics Swartjes JM, Th Arts NF. Fetal hiccups; characteristics
and relation to fetal heart rate. Eur $\mathcal{f}$ Obstet Gynecol Reprod
Biol $1989: 30: 209-16$.

17 Wigglesworth JS, Desai R. Effects of lung growth on cervical cord section in the rabbit fetus. Early Hum Dev 1979;3: cord sectic

18 Wigglesworth JS, Desai R. Is fetal respiratory function a major determinant of perinatal survival? Lancet 1982;i: $264-7$

19 Patrick J, Natale R, Richardson B. Patterns of human fetal breathing activity at 34 to 35 weeks gestational age. Am f Obstet Gynecol 1978;132:507-13.

20 Nijhuis JG, Martin CB Jr, Gommers S, Bouw S, Bots RSGM The rhythmicity of fetal breathing varies with behavioural tates in the human fetus. Early Hum Dev 1983;9:1-7.

21 van Vliet MAT, Martin CB Jr, Nijhuis JG, Prechtl HFR The relationship between fetal activity and behavioural states and fetal breathing movements in normal and growth-retarded fetuses. Am $\mathcal{f}$ Obstet Gynecol 1985;153: 582-8.

22 Richardson BS, Patrick JE, Abduljabbar H. Cerebral oxidative metabolism in the fetal lamb: relationship to electrocortical state. Am f Obstet Gymecol 1985;153:426-31. 